\title{
Hyaluronate lyase activity of Streptococcus suis serotype 2 and modulatory effects of hyaluronic acid on the bacterium's virulence properties
}

\author{
Bruno Haas ${ }^{1}$, Katy Vaillancourt ${ }^{1}$, Laetitia Bonifait ${ }^{1}$, Marcelo Gottschalk ${ }^{2,3}$ and Daniel Grenier ${ }^{1,2^{*}}$
}

\begin{abstract}
Background: Streptococcus suis serotype 2 is a major swine pathogen and zoonotic agent worldwide causing mainly meningitis and septicemia. Hyaluronate lyases are enzymes that degrade hyaluronic acid, a major constituent of animal tissues, and have been reported as virulence factors in various bacterial species. Since the hyaluronate lyase of $S$. suis has been considered ambiguously as a virulence factor, we screened 50 isolates from the three major clonal complexes found in North America (sequence type [ST] 1, ST25, and ST28) known to differ in their degree of virulence in order to link the presence or absence of this activity with the degree of virulence. Moreover, the effect of exogenous hyaluronic acid on S. suis virulence factor gene expression and the pro-inflammatory response of brain macrovascular endothelial cells (BMEC) was also investigated.
\end{abstract}

Results: We found that all but one ST1 isolates (high virulence) were devoid of hyaluronate lyase activity whereas all ST25 (intermediate virulence) and ST28 (low virulence) isolates possessed the activity. A 2 bp insertion was responsible for the lack of activity in ST1 strains. Since the most virulent isolates did not degrade hyaluronic acid, this tissue component may be found during the infectious process. Therefore, we investigated its effect on S. suis and host cells. Hyaluronic acid was found to modulate S. suis adhesion to BMEC, to increase S. suis virulence factor expression, and to enhance pro-inflammatory cytokine secretion by BMEC.

Conclusions: These findings suggest that $S$. suis hyaluronate lyase does not represent a critical virulence factor in its active form. However, exogenous hyaluronic acid that is likely to interact with S. suis and host cells during the course of infection appears to modulate several virulence determinants of the bacterium, in addition to promote inflammation.

Keywords: Adherence, Cytokines, Hyaluronate lyase, Hyaluronic acid, Streptococcus suis

\section{Background}

Streptococcus suis is an important swine pathogen worldwide responsible for substantial economic losses in the swine industry. Among the 29 known serotypes determined by the capsular antigenic composition, serotype 2 is predominant in swine infections, causing meningitis,

\footnotetext{
*Correspondence: daniel.grenier@greb.ulaval.ca

${ }^{1}$ Groupe de Recherche en Écologie Buccale (GREB), Faculté de Médecine Dentaire, Université Laval, 2420 Rue de la Terrasse, Quebec City, QC G1V 0A6, Canada

Full list of author information is available at the end of the article
}

pneumonia, septicemia, endocarditis and arthritis [1-3]. Moreover, S. suis is regarded as an emerging zoonotic agent, mostly in Asia, since it can infect humans in close contact with contaminated pigs or their byproducts. The general population in Asia is also at risk due to consumption of raw pork meat or blood [4]. Two S. suis outbreaks were reported in China in 1998 and 2005 that caused over 60 deaths [5]. S. suis is also the major cause of adult meningitis in Vietnam [6]. S. suis serotype 2 has been divided into clonal complexes composed of sequence types (STs) determined by multilocus sequence typing (MLST) [7]. 
Two dominant (ST25 and ST28) and one less frequent (ST1) STs were identified in North America, which differ in their virulence in a mouse infection model [8]. Strains belonging to ST1 are highly virulent whereas ST25 and ST28 isolates have an intermediate and low virulence, respectively.

To date, several virulence factors have been identified or proposed in S. suis $[9,10]$. The sialic-acid rich capsule is considered one of the most important virulence factors since acapsular mutants of $S$. suis were more susceptible to phagocytosis by macrophages and avirulent in animal models (piglet and mouse) [11, 12]. Additional virulenceassociated markers such as suilysin [13], extracellular factor [14], muraminidase-released protein [14], proteases $[15,16]$, and cell-wall anchored DNase [17] may also contribute to $S$. suis pathogenesis.

In 2004, Allen et al. identified a hyaluronate lyase produced by S. suis (serotype 7) [18]. Hyaluronate lyases degrade hyaluronic acid, a major constituent of the extracellular matrix [19] made of repeating disaccharide units of $\beta-1,4-D$-glucuronic acid- $\beta-1,3-N$-acetyl- $\beta$ D-glucosamine, by $\beta$-elimination at the $\beta-1,4$ glycosidic linkages leading to $\Delta 4,5$-unsaturated oligosaccharides [20]. Hyaluronate lyases produced by streptococci can play important roles in pathogenesis, from providing nutrients to the bacterium to enhancing the pathogen's spread into host tissues by degrading hyaluronic acid [21]. More specifically, the hyaluronate lyase produced by Streptococcus intermedius has been shown to contribute to bacterial detachment from biofilms, consequently enhancing its dissemination in the host [22]. Evidence have been brought that Streptococcus agalactiae hyaluronate lyase is involved in macrophage intracellular survival as well as regulation of pro-inflammatory cytokine expression [23]. The hyaluronate lyase produced by $S$. suis has been suggested as a virulence factor, although no direct link could be established by studying the distribution of active hyaluronate lyase among $S$. suis field strains. This latter study also showed that a number of strains of S. suis can display an inactive form of hyaluronate lyase due to a 2 bp insertion causing a shifted reading frame resulting in a truncated and inactive form of the protein [24]. A role assigned to $S$. suis hyaluronate lyase relates to degradation of hyaluronic acid generating oligosaccharides that were then processed by the bacterium as a carbon source [18]. A role of S. suis hyaluronate lyase in the pathogenesis of meningitis has been recently proposed by Wu et al. since the protein was able to interact with an angiogenin inhibitor, a property that may lead to an increased permeability of the blood brain barrier [25].

In group A streptococci (GAS), also known as Streptococcus pyogenes, the hyaluronate lyase has been shown to contribute to subcutaneous spread and growth of the pathogen [26]. However, in a more recent study, the GAS hyaluronate lyase has been found to be present in an inactive form in the most virulent strains [27]. Given the fact that GAS capsule is mainly constituted of hyaluronic acid, expression of a functional hyaluronate lyase may cause capsule degradation and therefore increase susceptibility of the pathogen to the host immune system. Consequently, Hynes et al. suggested that GAS hyaluronate lyase should be considered as an anti-virulence factor [27]. S. suis is also known to express a capsule $[9,10]$. However, its major constituent is sialic acid rather than hyaluronic acid as for S. pyogenes [27].

In the first part of this study, we investigated the distribution and genetic diversity of $S$. suis hyaluronate lyase among the three major STs of S. suis serotype 2 found in North America (ST1, ST25, ST28) that are known to differ in their degree of virulence, in order to provide an insight on the involvement of this protein in S. suis virulence. In the second part of the study, various aspects of the interaction between exogenous hyaluronic acid and S. suis were analyzed, including the effect on biofilm formation, adherence to brain microvascular endothelial cells (BMEC) and virulence factor gene expression. Lastly, since it has been shown that hyaluronic acid can regulate inflammation in host cells [28], we studied the effect of hyaluronic acid on pro-inflammatory cytokine secretion by BMEC.

\section{Methods}

\section{Bacterial strains and culture conditions}

Strains of S. suis serotype 2 used in this study as well as their corresponding ST and origin are listed in Table 1. Bacteria were routinely grown in Todd-Hewitt Broth (THB; BD-Canada, Mississauga, ON, Canada) at $37{ }^{\circ} \mathrm{C}$ unless specified otherwise.

\section{Plate assay for hyaluronate lyase activity}

Hyaluronate lyase activity was determined using the plate assay previously described by Smith and Willett [29]. Briefly, $10 \mu \mathrm{l}$ of overnight cultures of $S$. suis were spotted on Brain Heart Infusion (BHI; BD-Canada) agar plates supplemented with $0.04 \%(w / v)$ hyaluronic acid (SigmaAldrich Canada Co., Oakville, ON, Canada) and $1 \%(w / v)$ bovine serum albumin (BSA). Plates were then incubated for $24 \mathrm{~h}$ at $37^{\circ} \mathrm{C}$. Hyaluronate lyase activity was revealed by the addition of $2 \mathrm{M}$ acetic acid for 3 min which allows precipitation of the complex BSA/hyaluronic acid, the presence of a clear halo around bacterial growth area was indicative of hyaluronate lyase activity (Fig. 1).

\section{Gene amplification and sequencing}

In order to compare DNA sequences of the entire hyaluronate lyase gene found in S. suis serotype 2 strains 
Table 1 Sequence type, origin, type of infection, and hyaluronate lyase activity of S. suis serotype 2 isolates

\begin{tabular}{|c|c|c|c|c|}
\hline Strain & Sequence type & Origin & Type of infection & Hyaluronate lyase activity \\
\hline P156/00P6 & 1 & Argentina & Unknown & - \\
\hline P477/03P1 & 1 & Argentina & Meningitis & - \\
\hline P517/03P4 & 1 & Argentina & Meningitis & - \\
\hline P613/05P3 & 1 & Argentina & Unknown & - \\
\hline Prot 611/07 & 1 & Argentina & Unknown & - \\
\hline 31533 & 1 & France & Meningitis & - \\
\hline DAT229 & 1 & Japan & Endocarditis & - \\
\hline DAT261 & 1 & Japan & Pneumonia & - \\
\hline DAT264 & 1 & Japan & Meningitis & - \\
\hline $\mathrm{NIAH} 1143$ & 1 & Japan & Meningitis & - \\
\hline MNCM01 & 1 & Thailand & Endocarditis & - \\
\hline MNCM06 & 1 & Thailand & Meningitis & - \\
\hline MNCM16 & 1 & Thailand & Meningitis & - \\
\hline S735 & 1 & The Netherlands & Unknown & + \\
\hline $\mathrm{P} 1 / 7$ & 1 & United Kingdom & Meningitis & - \\
\hline MGGUS2 & 1 & USA & Meningitis & - \\
\hline MGGUS3 & 1 & USA & Meningitis & - \\
\hline 1043248 & 25 & Canada & Meningitis & + \\
\hline 1043629 & 25 & Canada & Pneumonia & + \\
\hline 1044423 & 25 & Canada & Unknown & + \\
\hline 1053253 & 25 & Canada & Pneumonia & + \\
\hline 1085543 & 25 & Canada & Meningitis & + \\
\hline 1102337 & 25 & Canada & Meningitis & + \\
\hline 1102864 & 25 & Canada & Septicemia & + \\
\hline LPH4 & 25 & Thailand & Septicemia & + \\
\hline LPH12 & 25 & Thailand & Septic shock & + \\
\hline MNCM04 & 25 & Thailand & Meningitis & + \\
\hline MNCM10 & 25 & Thailand & Septicemia & + \\
\hline MNCM24 & 25 & Thailand & Endocarditis & + \\
\hline MNCM26 & 25 & Thailand & Endocarditis & + \\
\hline MNCM51 & 25 & Thailand & Septicemia & + \\
\hline MGGUS4 & 25 & USA & Meningitis & + \\
\hline MGGUS5 & 25 & USA & Unknown & + \\
\hline 1054471 & 28 & Canada & Meningitis & + \\
\hline 1057906 & 28 & Canada & Meningitis & + \\
\hline 1084708 & 28 & Canada & Unknown & + \\
\hline 1088563 & 28 & Canada & Meningitis & + \\
\hline 1097205 & 28 & Canada & Meningitis & + \\
\hline 90-1330 & 28 & Canada & Healthy pig & + \\
\hline DAT242 & 28 & Japan & Meningitis & + \\
\hline DAT245 & 28 & Japan & Meningitis & + \\
\hline DAT246 & 28 & Japan & Septicemia & + \\
\hline DAT251 & 28 & Japan & Unknown & + \\
\hline DAT292 & 28 & Japan & Unknown & + \\
\hline MNCM43 & 28 & Thailand & Endocarditis & + \\
\hline MGGUS9 & 28 & USA & Endocarditis & + \\
\hline MGGUS10 & 28 & USA & Pneumonia & + \\
\hline MGGUS11 & 28 & USA & Pneumonia & + \\
\hline MGGUS12 & 28 & USA & Pneumonia & + \\
\hline MGGUS13 & 28 & USA & Meningitis & + \\
\hline
\end{tabular}




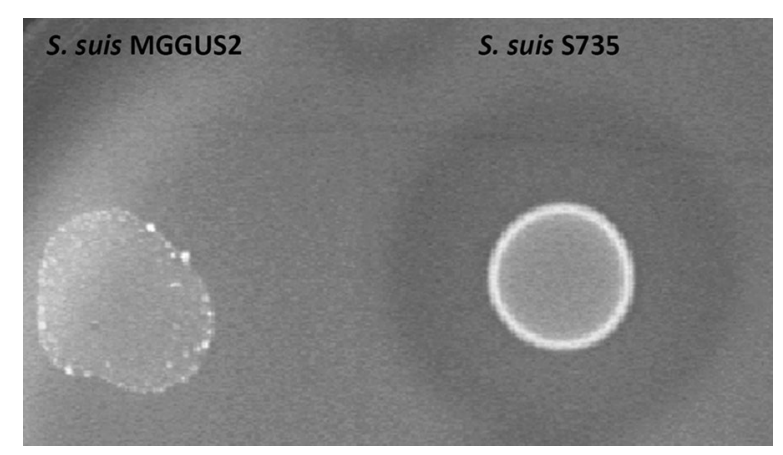

Fig. 1 Hyaluronate lyase activity of S. suis MGGUS2 (HylA -, left) and S. suis $\mathrm{S} 735$ (HylA +, right), as determined using the plate assay

belonging to different STs, amplification of overlapping fragments was performed by PCR using primers designed with Primer3Plus [30] and listed in Table 2. Amplicons were then sequenced, the full hylA genes were assembled and then compared to the hyaluronate lyase gene previously identified in S. suis serotype 7 (accession number AJ3088328) [18] using BioEdit (http://www.mbio.ncsu.edu/BioEdit/bioedit.html) and the NCBI database (http://blast.ncbi.nlm.nih.gov/Blast. cgi).

\section{Effect of hyaluronic acid on biofilm formation by S. suis}

An overnight culture of $S$. suis P1/7 (ST1, negative for hyaluronate lyase activity) was diluted to an optical density at $660 \mathrm{~nm}\left(\mathrm{OD}_{660}\right)$ of 0.1 in THB supplemented or not with hyaluronic acid (Sigma-Aldrich Canada Co.; 0.01-1 mg/ml). Two hundred microliters of the bacterial suspension were seeded into wells of a 96-well tissue culture microplate. After incubation at

Table 2 Primers used for the amplification of hyaluronate lyase gene in $\mathrm{S}$. suis strains

\begin{tabular}{ll}
\hline Primer name & Sequence $\left(\mathbf{5}^{\prime} \mathbf{- 3}^{\mathbf{\prime}} \mathbf{)}\right.$ \\
\hline HYLA5587F & CCCGCATAAAAAGGAAAGGAAACAC \\
HYLA5614Fi & ATGGGATTTTTTATCAGTCAAAGCA \\
HYLA5916R & TTCTTTTGTCGCCTGAACAGCTTGC \\
HYLA6414 & CGAATACGTAGAAAAGACACCAGAA \\
HYLA6414R & TTCTGGTGTTTTTCTACGTATTCG \\
HYLA6722R & TCGTTAGATTGGTAGCGTTTATTGG \\
HYLA7213 & AGACGGATTTGAGCAAGGTGGCCAT \\
HYLA7516R & GTCATATCCATCAGTTCACCGCGAA \\
HYLA8014 & TTACCGGGAACGACAACGACCAAGG \\
HYLA8315R & TCTGTCACTACTTCTTTGGACAAAT \\
HYLA8792 & CCGTAAAGAATGGCATTGAGTTGAC \\
HYLA9109Ri & AGGAGTGCGATCTGCGACACTTGAA \\
HYLA9138R & CGCTCCCCCTTCACCCACGAATTC \\
\hline
\end{tabular}

$37^{\circ} \mathrm{C}$ for $24 \mathrm{~h}, \mathrm{~S}$. suis growth was monitored by recording the $\mathrm{OD}_{660}$. The medium and planktonic bacteria were then aspirated and biofilms were washed three times with $50 \mathrm{mM}$ phosphate-buffered saline (PBS; $\mathrm{pH}$ 7.2). Biofilms were then stained with $100 \mu \mathrm{l}$ of $0.04 \%$ crystal violet for $10 \mathrm{~min}$. Three washing steps were performed with PBS to remove the excess of dye and plates were left to dry for $2 \mathrm{~h}$ at $37{ }^{\circ} \mathrm{C}$ before adding $100 \mu \mathrm{l} /$ well of $95 \%$ ethanol and shaking for $10 \mathrm{~min}$ to release the stain. The absorbance at $550 \mathrm{~nm}\left(\mathrm{~A}_{550}\right)$ was then recorded with a xMark microplate spectrophotometer (Bio-Rad Laboratories, Mississauga, ON, Canada).

\section{Effect of hyaluronic acid on adherence of $S$. suis to BMEC}

The human BMEC cell line used in this study has been previously described [31]. Cells were grown in RPMI 1640 medium (Life Technologies Inc., Burlington, ON, Canada) supplemented with $10 \%(\mathrm{v} / \mathrm{v})$ heatinactivated fetal bovine serum (Life Technologies Inc.), $10 \%(\mathrm{v} / \mathrm{v}) \mathrm{Nu}-$ Serum IV supplement (Becton-Dickinson Biosciences, Bedford, MA, USA), $2.05 \mathrm{mM} \mathrm{L-glu-}$ tamine, and $1 \%$ penicillin-streptomycin at $37{ }^{\circ} \mathrm{C}$ in a $5 \% \mathrm{CO}_{2}$ atmosphere. One hundred microliters of a BMEC suspension $\left(10^{6}\right.$ cells $\left./ \mathrm{ml}\right)$ were seeded into wells of a 96-well black wall microplate with clear bottom and incubated overnight at $37{ }^{\circ} \mathrm{C}$ with $5 \% \mathrm{CO}_{2}$. An overnight culture of S. suis P1/7 (ST1, negative for hyaluronate lyase activity) was centrifuged at $10,000 \times g$ for $20 \mathrm{~min}$ at $4{ }^{\circ} \mathrm{C}$ and washed once in PBS. Bacteria were then resuspended in bicarbonate buffer $(0.5 \mathrm{M}$ $\left.\mathrm{NaHCO}_{3}, \mathrm{pH} 8\right)$ to an $\mathrm{OD}_{660}=1$, and fluorescein isothiocyanate (FITC) was added to a final concentration of $0.03 \mathrm{mg} / \mathrm{ml}$. Labeling of the bacteria was performed at $37{ }^{\circ} \mathrm{C}$ for $30 \mathrm{~min}$. In the meantime, BMEC were washed 3 times with PBS pH 7.2 and $100 \mu$ l of culture medium supplemented with hyaluronic acid (SigmaAldrich Canada Co.; 0.1, 0.5 and $1 \mathrm{mg} / \mathrm{ml}$ ) was added. Cells were then incubated for $30 \mathrm{~min}$ at $37{ }^{\circ} \mathrm{C}$ in the presence of $5 \% \mathrm{CO}_{2}$. FITC-labelled bacteria were washed 3 times with PBS and $100 \mu$ of this suspension was added to the BMEC monolayers to a final multiplicity of infection (MOI) of 200. The microplate was then subjected to a low-speed centrifugation $(5 \mathrm{~min}$ at $200 \times g$ ) to allow contact of bacteria with BMEC, and incubated at $37{ }^{\circ} \mathrm{C}$ in the presence of $5 \% \mathrm{CO}_{2}$ for $2 \mathrm{~h}$. Wells were washed twice with PBS and $200 \mu \mathrm{l}$ PBS was added prior to record fluorescence using a Synergy 2 microplate reader (BioTek Instruments Inc, Winooski, VT, USA) with an excitation wavelength of $485 \mathrm{~nm}$ and an emission wavelength of $528 \mathrm{~nm}$. Percentage of adherence was calculated using the control value (no hyaluronic acid) as $100 \%$. 


\section{Effect of hyaluronic acid on virulence factor gene expression by $S$. suis}

S. suis $\mathrm{P} 1 / 7$ (ST1, negative for hyaluronate lyase activity) was grown in THB supplemented with hyaluronic acid (Sigma-Aldrich Canada Co.; 0.625, 1.25 and $2.5 \mathrm{mg} / \mathrm{ml}$ ) for $6 \mathrm{~h}$ in three independent cultures. Total RNA was extracted using the RNeasy minikit (Qiagen Inc., Mississauga, ON, Canada). The RNA quality was controlled using the Experion RNA StdSens Analysis kit (Bio-Rad Laboratories). Purity and quantity of the RNA were measured using Nanodrop (Thermo Fisher Scientific, Wilmington, DE, USA). Samples were then diluted in nuclease-free water to a final concentration of $100 \mathrm{ng} / \mu \mathrm{l}$ and cDNA were produced using iScript R-T Supermix for RT-qPCR (Bio-Rad Laboratories) according to the manufacturer's protocol. Gene expression of five putative virulence factors (capsule [cps2J], muraminidase-released protein $[m r p]$, extracellular protein factor [epf], suilysin [sly] and cell-wall anchored DNase $[\operatorname{ssn} A]$ ) was recorded using the CFX96 Real-Time System C1000 Thermal Cycler (Bio-Rad Laboratories) with the $\mathrm{IQ}^{\mathrm{TM}}$ SYBR Green fluorophore (Bio-Rad Laboratories) according to the manufacturer's protocols, using the $16 \mathrm{~S}$ rRNA as internal control for data normalization as it is routinely used for gene expression quantification in $S$. suis [32]. Primers, purchased from Life Technologies Inc, are listed in Table 3. To validate the specificity for each primer pair, melting curves were analyzed and migration of PCR products on $1 \%$ agarose gel $45 \mathrm{~min}$ at $100 \mathrm{~V}$ was performed. Amplicons migrated to their expected size of 123 bp (16S rRNA), 198 bp (cps2J), $216 \mathrm{bp}$ (mrp), $162 \mathrm{bp}$ (epf), $97 \mathrm{bp}(s l y)$ and $390 \mathrm{bp}$ $(\operatorname{ssn} A)$ (data not shown).

Table 3 Primers used for the determination of virulence factor gene expression in S. suis P1/7

\begin{tabular}{|c|c|c|}
\hline Gene & Primer sequence $\left(5^{\prime}-3^{\prime}\right)$ & $\begin{array}{l}\text { Expected } \\
\text { amplicon } \\
\text { size (bp) }\end{array}$ \\
\hline $16 \mathrm{~S}$ rRNA & $\begin{array}{l}\text { Forward: TAGGGTTTCTCTTCGGAGCATCG } \\
\text { Reverse: AACTGAATGATGGCAACT }\end{array}$ & 123 \\
\hline $\operatorname{cps} 2 J$ & $\begin{array}{l}\text { Forward: AGAGTGTTTAGATAGCATTATTTCC } \\
\text { Reverse: TAATTTGCTGTGCTATTTTTGATAC }\end{array}$ & 198 \\
\hline$m r p$ & $\begin{array}{l}\text { Forward: TATCAGATTAATACAACTCGTTACG } \\
\text { Reverse: ATATTGTACTGTATCCTTTTGAACC }\end{array}$ & 216 \\
\hline epf & $\begin{array}{l}\text { Forward: CTAAACGTAACTTGGAATTTGTAAG } \\
\text { Reverse: AGCCATAAGTAAGATTATTTGATCC }\end{array}$ & 162 \\
\hline sly & $\begin{array}{l}\text { Forward: TTGAATATTGACATGAAGATTGCGA } \\
\text { Reverse: AAGCTGGAGAAGTTTGGGAACC }\end{array}$ & 97 \\
\hline $\operatorname{ssn} A$ & $\begin{array}{l}\text { Forward: GCTGACTATCGCTTCTTATAACATT } \\
\text { Reverse: GTCGATTCGGCCTAGGCTGAGATTG }\end{array}$ & 390 \\
\hline
\end{tabular}

Effect of hyaluronic acid on secretion of pro-inflammatory cytokines by BMEC

Two $\mathrm{ml}$ of a suspension of BMEC $\left(10^{6}\right.$ cells $\left./ \mathrm{ml}\right)$ in the culture medium described above were seeded into each well of a 6-well microplate and incubated overnight at $37{ }^{\circ} \mathrm{C}$ in a $5 \% \mathrm{CO}_{2}$ atmosphere. The medium was then aspirated and replaced with fresh culture medium supplemented with hyaluronic acid (Sigma-Aldrich Canada Co.; 0.1, 0.5, 1, and $2 \mathrm{mg} / \mathrm{ml}$ ). Hyaluronic acid treatments were performed for $24 \mathrm{~h}$ at $37^{\circ} \mathrm{C}$ prior to collect culture supernatants for quantification of interleukin-1 $\beta$ (IL-1 $\beta$ ), tumor necrosis factor- $\alpha$ (TNF- $\alpha$ ), interleukin-6 (IL-6) and interleukin-8 (CXCL8) by enzyme-linked immunosorbent assays (ELISA) (eBioscience Inc., San Diego, CA, USA) according to the manufacturer's instructions.

\section{Statistical analysis}

All assays were performed in triplicate and the means \pm standard deviations were calculated. Differences were analyzed for statistical significance using the Student's $t$ test and were considered significant at $p<0.05$.

\section{Results}

Distribution of hyaluronate lyase activity among S. suis STs Fifty S. suis serotype 2 strains (17 ST1, 16 ST25, and 17 ST28) were tested for hyaluronate lyase activity on BHI plates supplemented with BSA and hyaluronic acid. As shown in Fig. 1, positive hyaluronate lyase activity resulted in the formation of a clear halo around the bacterial growth. It was found that sixteen out of seventeen isolates belonging to ST1, which comprises the most virulent strains, were devoid of hyaluronate lyase activity (Table 1), whereas all ST25 (intermediate virulence) and ST28 (low virulence) isolates possessed the activity. $S$. suis S735 was the only ST1 isolate possessing hyaluronate lyase activity.

\section{Comparative analysis of $S$. suis hyaluronate lyase gene}

Given the differences observed in hyaluronate lyase activity of strains belonging to different STs, the hyaluronate lyase gene of nine ST1, three ST25 and three ST28 strains was amplified. As we were unable to obtain a proper amplification of the full gene due to non-specific amplification, PCR of overlapping fragments were performed. The resulting products were then sequenced and the full gene was then assembled for analysis. Comparison of the nucleic acid sequences between STs using S. suis serotype 7 hylA gene previously described as reference gene (accession number AJ3088328) [18] showed the presence of four conserved insertions that occurred in ST1 strains (Fig. 2): a 21 bp insertion starting at position 192, a 3 bp insertion starting at position 330, a 2 bp insertion at 


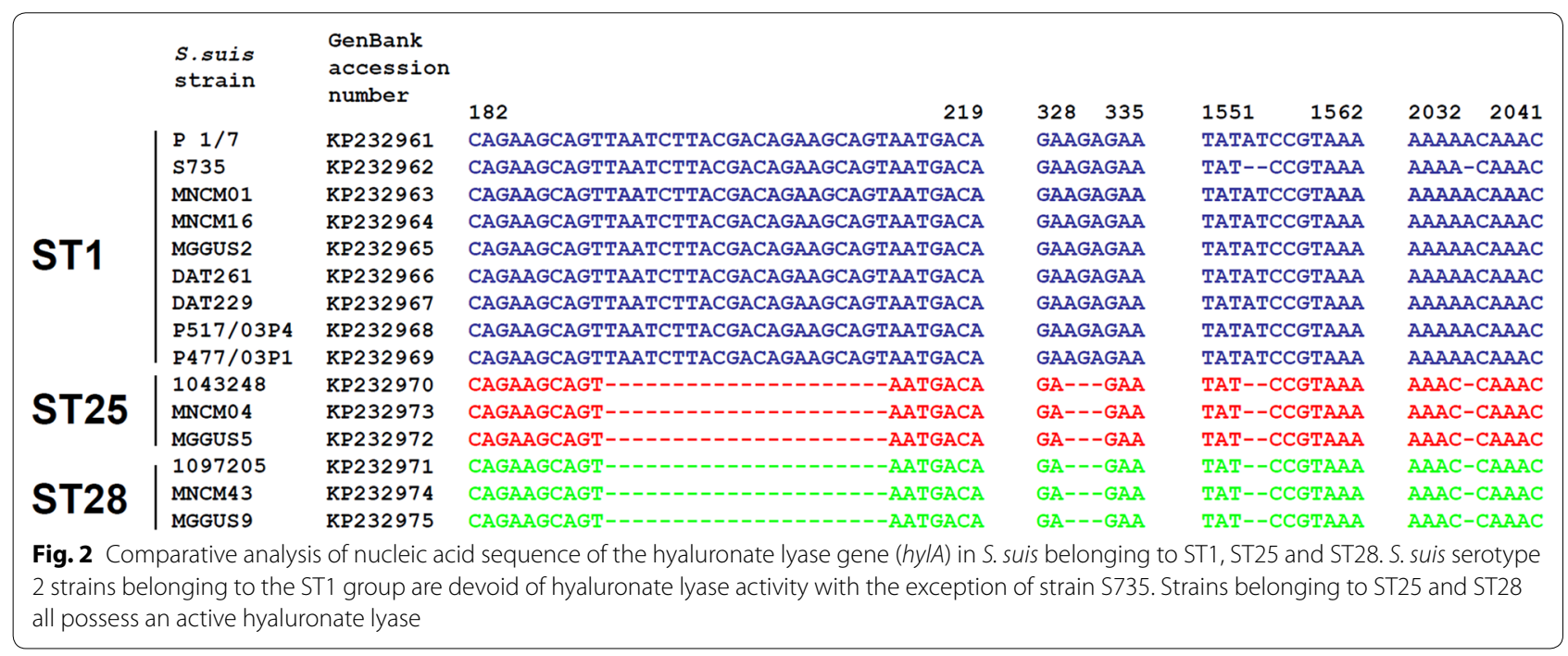

position 1554-1555 and a single bp insertion at position 2036. Interestingly, the only ST1 strain (S735) that possessed hyaluronate lyase activity did not display the two last insertions in the hyaluronate lyase gene.

\section{Effect of hyaluronic acid on biofilm formation, adherence to $B M E C$, and virulence factor gene expression in S. suis P1/7}

Given that the above data indicated that the most virulent strains of $S$. suis (ST1) do not possess hyaluronate lyase activity and consequently are likely to interact with hyaluronic acid during the course of infection, we then investigated the effect of this glycosaminoglycan on biofilm formation, adherence to BMEC, and virulence factor gene expression in S. suis P1/7 (ST1, negative for hyaluronidase activity).

The biofilm assay was performed in a microplate model following growth of $S$. suis in THB supplemented with hyaluronic acid, prior to crystal violet staining. Growth of $S$. suis was not affected by the presence of hyaluronic acid at concentrations of $0.01-0.1 \mathrm{mg} / \mathrm{ml}$, as determined by recording the $\mathrm{OD}_{660}$ following overnight incubation. However, in the presence of high concentrations of hyaluronic acid $(0.5$ and $1 \mathrm{mg} / \mathrm{ml})$, bacterial growth was reduced (Fig. 3a). In regard to biofilm formation, no significant effect of hyaluronic acid was observed at $0.01 \mathrm{mg} / \mathrm{ml}$, whereas at $0.05 \mathrm{mg} / \mathrm{ml}$ of hyaluronic acid, biofilm formation was significantly reduced. Hyaluronic acid at concentrations of 0.1 and $0.5 \mathrm{mg} / \mathrm{ml}$ increased biofilm formation by S. suis $\mathrm{P} 1 / 7$. At the highest concentration tested of hyaluronic acid $(1 \mathrm{mg} / \mathrm{ml})$, biofilm formation by $S$. suis was attenuated and may result from the reduced bacterial growth (Fig. $3 \mathrm{~b}$ ).

To investigate the effect of hyaluronic acid on $S$. suis adherence to BMEC, FITC-labelled bacteria were
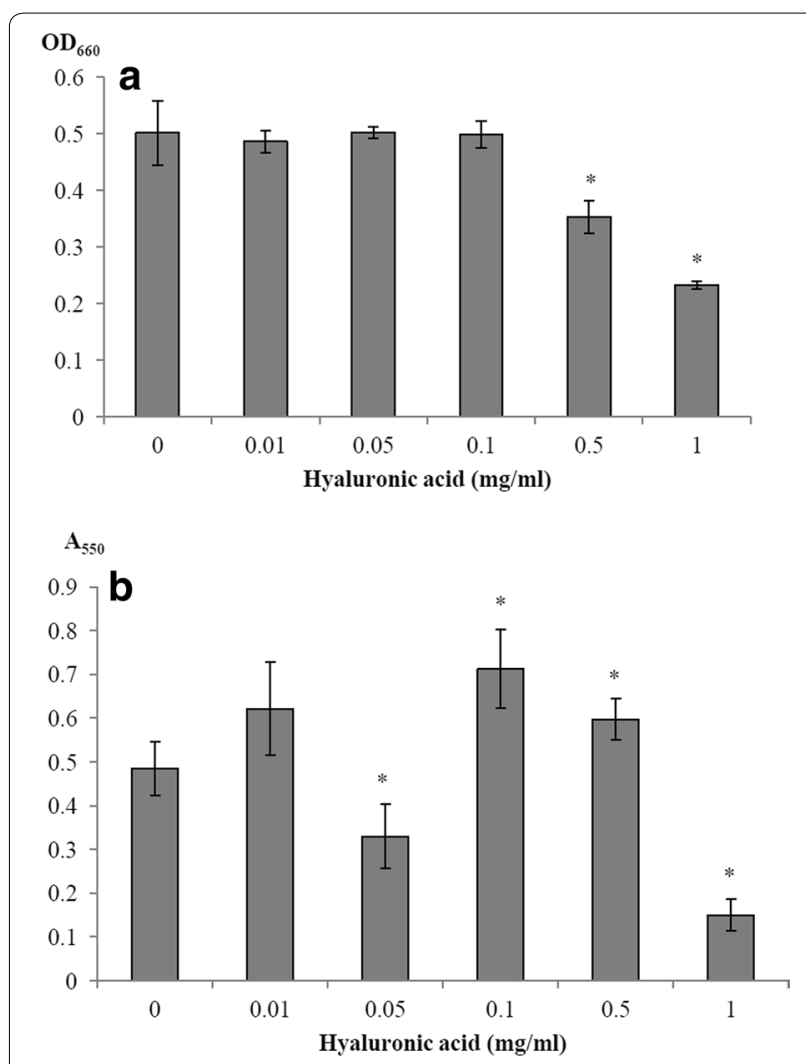

Fig. 3 Effect of hyaluronic acid on S. suis P1/7 growth (a) and biofilm formation (b). * Significantly different from control at $p<0.05$

incubated with a monolayer of cells in the presence of hyaluronic acid at concentrations ranging between 0.1 and $1 \mathrm{mg} / \mathrm{ml}$. Adherence of $S$. suis relative to control (without hyaluronic acid) is reported in Fig. 4. An increased adherence $(\sim 35 \%)$ was observed in the presence of hyaluronic 


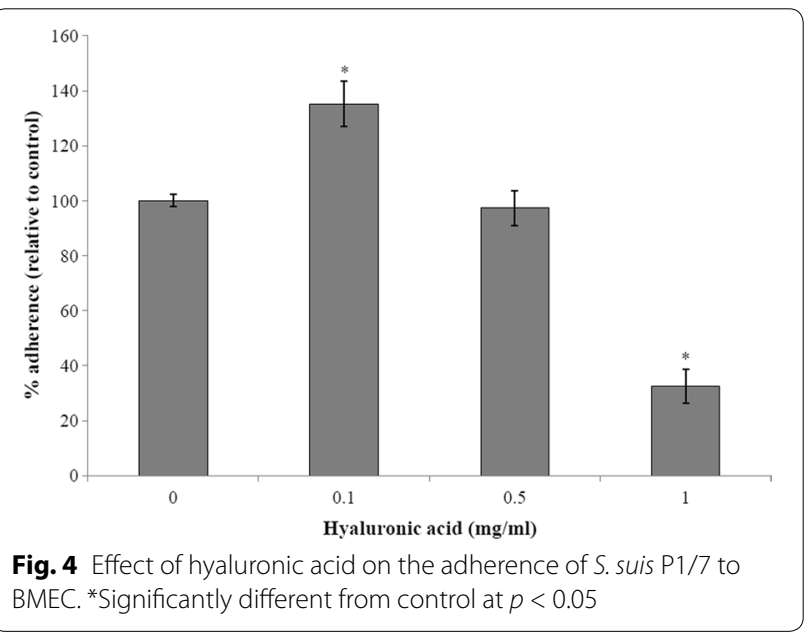

acid at a concentration of $0.1 \mathrm{mg} / \mathrm{ml}$ while the highest concentration of hyaluronic acid $(1 \mathrm{mg} / \mathrm{ml})$ caused an inhibition of adherence of $S$. suis to BMEC. More specifically, adherence of S. suis P1/7 decreased by $68 \%$.

The effect of hyaluronic acid on gene expression of five virulence-associated markers (capsule [cps2J], extracellular protein factor [epf], muraminidase-released protein $[m r p]$, suilysin $[s l y]$ and DNase $[s s n A])$ in S. suis P1/7 was determined by RT-qPCR using $16 \mathrm{~S}$ rRNA as reference gene. Hyaluronic acid at 0.625 and $1.25 \mathrm{mg} / \mathrm{ml}$ increased cps expression by a factor 1.3 and 1.8, respectively (Fig. 5a). An increase in epf expression was also observed in the presence of hyaluronic acid (Fig. 5b); the highest increase (1.65-fold) was observed with $1.25 \mathrm{mg} /$ $\mathrm{ml}$ of hyaluronic acid. In the presence of $\leq 2.5 \mathrm{mg} / \mathrm{ml}$ hyaluronic acid, the expression of $m r p$ increased, reaching a maximum of 2 -fold increase at $1.25 \mathrm{mg} / \mathrm{ml}$ (Fig. 5c). The expression of sly was 1.6- and 1.7-fold higher in the presence of 1.25 and $2.5 \mathrm{mg} / \mathrm{ml}$ hyaluronic acid, respectively, compared to control (Fig. 5d). Lastly, the expression of $\operatorname{ssn} A$ was 1.5- and 1.7-fold higher in the presence of hyaluronic acid at 0.625 and $1.25 \mathrm{mg} / \mathrm{ml}$ hyaluronic acid, respectively (Fig. 5e).

\section{Effect of hyaluronic acid on pro-inflammatory cytokine secretion by BMEC}

BMEC were treated with hyaluronic acid $(18 \mathrm{~h})$ at concentrations ranging from 0.1 to $2 \mathrm{mg} / \mathrm{ml}$ prior to determine pro-inflammatory cytokine (IL-6, CXCL-8, IL-1 $\beta$ and TNF- $\alpha$ ) secretion by ELISA. While no production of IL- $1 \beta$ and TNF- $\alpha$ was observed (data not shown), levels of IL- 6 and CXCL-8 increased in a dose-dependent fashion as reported in Fig. 6a, b, respectively. More specifically, when hyaluronic acid was added at $2 \mathrm{mg} / \mathrm{ml}$, IL-6 and CXCL-8 secretion was 3.6 and 12.8 times higher than the control, respectively.

\section{Discussion}

In 2004 , a hyaluronate lyase $(130 \mathrm{kDa})$ produced by the swine pathogen S. suis (serotype 7) has been identified and proposed as a virulence factor [18]. The fact that not all virulent strains expressed an active form of the enzyme suggested that $S$. suis hyaluronate lyase should not be considered as an essential virulence factor [24]. In this study, we focused on strains of S. suis serotype 2, which represents the most commonly isolated serotype from sick animals. In order to further investigate the relationship between expression of active hyaluronate lyase and virulence, we used $S$. suis serotype 2 strains belonging to the three clonal complexes present in North America, known as ST1, ST25 and ST28. It has been previously reported that these STs differ in their degree of virulence in a mouse infection model, ST1 isolates possess a high virulence whereas ST25 and ST28 isolates have an intermediate and low virulence, respectively [8]. While all ST25 and ST28 strains possessed hyaluronate lyase activity, sixteen out of seventeen isolates belonging to ST1 were devoid of activity. Interestingly, the only ST1 isolate positive for hyaluronate lyase activity (S. suis S735) has been previously reported to be only weakly virulent in piglets [33]. From the above data, an inverse correlation between virulence and the presence of hyaluronate lyase activity can be suggested.

Genetic analysis of the hyaluronate lyase gene of ST1, ST25 and ST28 strains showed the presence of four conserved insertions in strains belonging to ST1. The 2 bp insertion at position 1554-1555 is responsible for a shifted reading frame leading to an early stop codon resulting in a truncated form of the hyaluronate lyase in ST1 isolates, thus explaining the lack of hyaluronate lyase activity in these strains. Interestingly, it has been shown that this truncated protein $(53 \mathrm{kDa})$ was able to interact with an angiogenin inhibitor [25]. Such an interaction could inactivate the angiogenin inhibitor, thus promoting angiogenesis and vascular permeability that could allow S. suis to cross the blood-brain barrier and cause meningitis. It can be speculated that the loss of hyaluronate lyase activity in the most virulent strains of $S$. suis could have resulted in an increased bacterial virulence through such a mechanism.

Given that S. suis ST1 strains that do not produce active hyaluronate lyase are likely to interact with host hyaluronic acid in vivo, we then investigated the effects of this glycosaminoglycan on some biological properties of S. suis P1/7, a reference ST1 strain. Since the hyaluronic acid-rich capsule of $S$. pyogenes has been described as important for biofilm formation, as it could be involved in intercellular adherence between bacteria [34], we evaluated whether supplementing the culture medium with exogenous hyaluronic acid could induce 

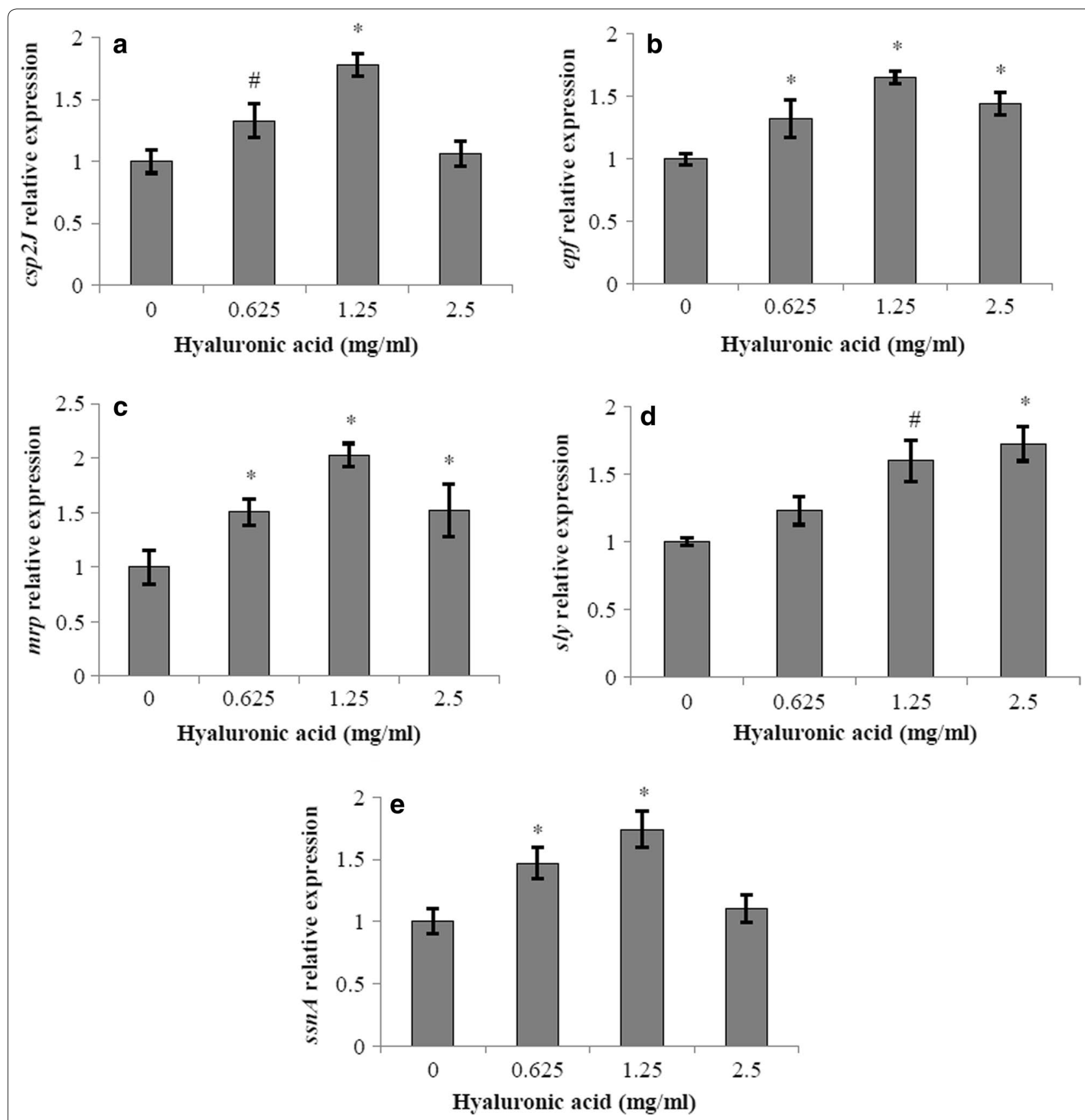

Fig. 5 Effect of hyaluronic acid on virulence factor gene expression by S. suis P1/7. Relative gene expression of $\operatorname{cps} 2 J(\mathbf{a}), \operatorname{epf}(\mathbf{b}), \operatorname{mrp}(\mathbf{c}), \operatorname{sly}(\mathbf{d}), \operatorname{ssnA}$ (e). Significantly different from control at ${ }^{\#} p<0.05$; ${ }^{*} p<0.01$

biofilm formation by $S$. suis, for which most strains cannot form a biofilm in a basal culture medium [35]. Our data revealed that hyaluronic acid at low concentration $(0.05 \mathrm{mg} / \mathrm{ml})$ reduced biofilm formation whereas higher concentrations $(0.1$ and $0.5 \mathrm{mg} / \mathrm{ml})$ stimulated biofilm formation. These observations suggest that at a concentration of $0.05 \mathrm{mg} / \mathrm{ml}$, hyaluronic acid could interfere with intercellular adhesion of S. suis or exopolysaccharide secretion. It was also found that high concentration of hyaluronic acid $(1 \mathrm{mg} / \mathrm{ml})$ inhibits the ability of S. suis to grow and produce a biofilm, probably due to the high osmotic pressure created.

It has been reported that at the early step of the pathogenic process of meningitis, S. suis adheres to BMEC 

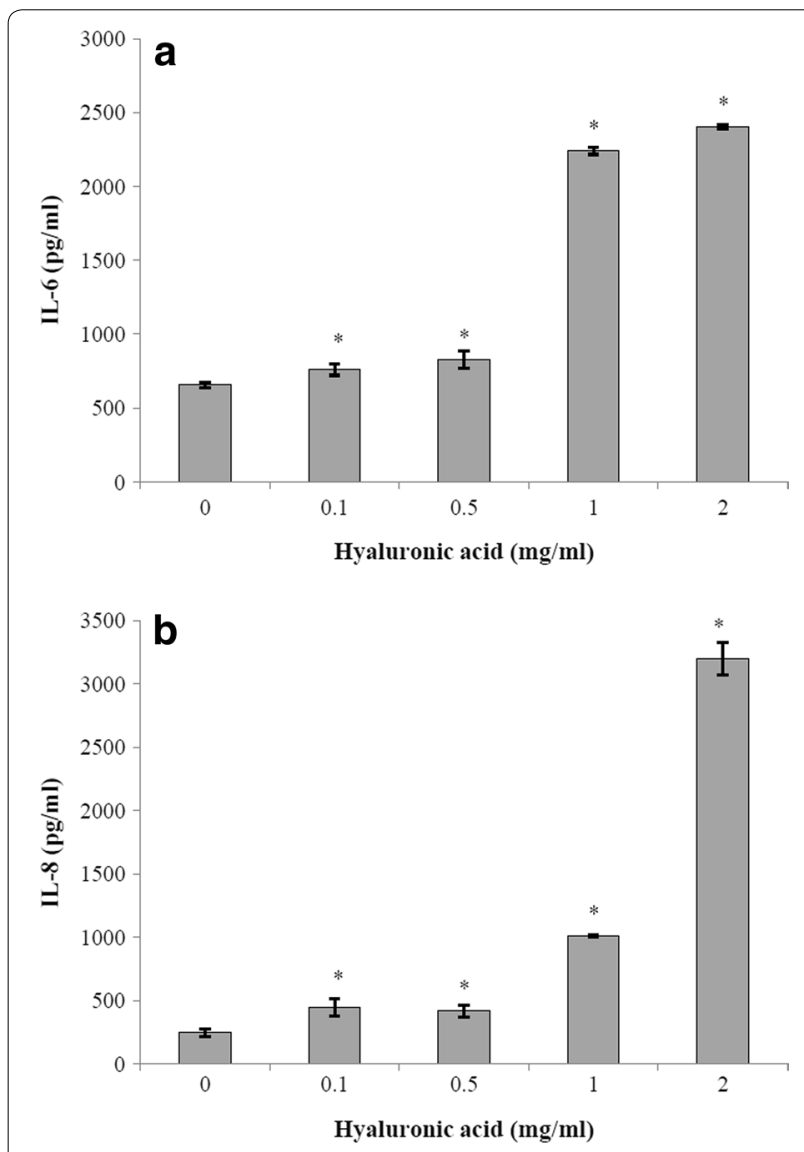

Fig. 6 Effect of hyaluronic acid on the secretion of IL-6 (a) and IL-8 $(\mathrm{CXCL}-8)(\mathbf{b})$ by BMEC. * Significantly different from control at $p<0.05$

in order to facilitate migration of bacteria through the blood-brain barrier [36]. In this regard, we then evaluated the effect of exogenous hyaluronic acid on the adherence of S. suis P1/7 (ST1) to BMEC. In the presence of hyaluronic acid at a low concentration $(0.1 \mathrm{mg} / \mathrm{ml})$, bacterial adherence was increased, while at a high concentration $(1 \mathrm{mg} / \mathrm{ml})$, the adherence of $S$. suis to BMEC was significantly decreased. Using FITC-labeled hyaluronic acid, it was found that hyaluronic acid preferentially binds to BMEC and not to bacteria (Additional file 1: Figure S1). From the above observations, it may be suggested that at high concentrations, binding of hyaluronic acid to BMEC could lead to a decreased accessibility of receptors for S. suis, resulting in a lower bacterial adherence to BMEC. However, at low concentrations, the cell surface charge of the BMEC may be modified by hyaluronic acid, resulting in increased bacterial adherence. Interestingly, hyaluronic acid has been previously reported to modulate the adherence of Mycobacterium tuberculosis to lung epithelium [37]. Our results are thus in agreement with these previous observations, especially that hyaluronic acid at high concentrations inhibits bacterial adherence.
However, the molecular mechanisms of S. suis adherence to cells via exogenous hyaluronic acid remains to be characterized.

It has been shown that growing S. pyogenes in the presence of hyaluronic acid resulted in an increased expression of several virulence factors, including the M1 protein and a collagen-like surface protein, that play key roles in the pathogenesis of GAS infections [38]. Growing S. suis $\mathrm{P} 1 / 7$ in hyaluronic acid-supplemented culture medium was found to increase the expression of several virulenceassociated markers such as the cell wall-anchored DNase, suilysin, MRP, EPF and capsule. Consequently, it can be hypothesized that the virulence of $S$. suis can be modulated by the presence of hyaluronic acid under in vivo conditions. Given the fact that $S$. suis P1/7 does not express an active form of hyaluronate lyase, the changes observed in virulence-associated markers gene expression can be linked with an adaptation to an osmotic or acidic stress in the culture medium supplemented with hyaluronic acid.

Hyaluronic acid from the host is likely to be present during infections caused by S. suis ST1 isolates, considering that they do not produce an active hyaluronate lyase. Hyaluronic acid has been reported to possess pro- and anti-inflammatory properties depending on the size of the molecule [28]. In our study, stimulation of BMEC with hyaluronic acid was associated with a proinflammatory effect. More specifically, hyaluronic acid was found to dose-dependently increase the secretion of IL-6 and CXCL-8, two major inflammatory mediators. In a previous study, it has been reported that hyaluronic acid can modulate pro-inflammatory cytokines secretion in human uterine fibroblasts by interacting with the CD44 receptor [39]. Our observations suggest a lower inflammatory potential for strains possessing an active hyaluronate lyase. Indeed, these isolates could degrade hyaluronic acid in the extracellular matrix thus decreasing pro-inflammatory cytokines secretion by host cells.

\section{Conclusions}

Our study brought evidence that hyaluronate lyase in its full length and active form does not represent a key virulence factor for S. suis. Indeed, hyaluronate lyase appears to be active only in isolates of S. suis serotype 2 belonging to the less virulent STs (ST25 and ST28). Considering that the S. suis P1/7 hyaluronate lyase (truncated and inactive form) has been shown to interact with an angiogenin inhibitor [25], it can be suggested that the highly conserved $2 \mathrm{bp}$ insertion in the hyaluronate lyase gene could lead to more invasive isolates by enhancing vascular permeability of the blood-brain barrier leading to the development of meningitis. Since Wu et al. [25] mainly focused their study on the truncated form of hyaluronate 
lyase, no data are available on the interaction of the active form of hyaluronate lyase with the angiogenin inhibitor, a phenomenon that may be important in the endothelial dysregulation associated with meningitis. Given that strains of S. suis belonging to ST1, the most virulent ST, do not degrade hyaluronic acid, we investigated the effect of this glycosaminoglycan on S. suis and host cells. While hyaluronic acid did not promote biofilm formation, it was found to modulate $S$. suis adhesion to BMEC. Moreover, hyaluronic acid increased virulence factor gene expression in S. suis and enhanced pro-inflammatory cytokine secretion by BMEC.

\section{Additional file}

Additional file 1: Figure S1. Binding of hyaluronic acid to BMEC and S. suis P1/7 (bacteria). No competition: FITC-labeled hyaluronic acid used alone; Competition: binding competition between FITC-labeled hyaluronic acid and non-labeled hyaluronic acid. Lower fluorescence in the competition assay is the result of binding of non-labeled hyaluronic acid to BMEC.

\section{Authors' contributions}

$\mathrm{BH}$ was the main experimentator for this study while LB carried out the screening of the S. suis stains for hyaluronate lyase activity. KV was in charge of the molecular biology assays and analysis. MG provided the S. suis strains. DG supervised and conceived this study. $\mathrm{BH}$ prepared the manuscript under the supervision of DG. All authors read and approved the final manuscript.

\section{Author details}

${ }^{1}$ Groupe de Recherche en Écologie Buccale (GREB), Faculté de Médecine Dentaire, Université Laval, 2420 Rue de la Terrasse, Quebec City, QC G1V 0A6, Canada. ${ }^{2}$ Centre de Recherche en Infectiologie Porcine et Avicole (CRIPA), Fonds de Recherche du Québec-Nature et Technologies (FRQNT), Saint-Hyacinthe, QC, Canada. ${ }^{3}$ Groupe de Recherche sur les Maladies Infectieuses du Porc (GREMIP), Faculté de Médecine Vétérinaire, Université de Montréal, Saint-Hyacinthe, QC, Canada.

\section{Acknowledgements}

We are grateful to K.S. Kim (John Hopkins University, School of Medicine) for providing the human BMEC. This work was supported by the Natural Sciences and Engineering Research Council of Canada. $\mathrm{BH}$ is recipient of a scholarship from the Swine and Poultry Infectious Disease Research Center (CRIPA).

\section{Competing interests}

The authors declare that they have no competing interests.

Received: 3 August 2015 Accepted: 12 November 2015

Published online: 26 November 2015

\section{References}

1. Gottschalk M. Streptococcosis. In: Karriger LRA, Schwartz KJ, Stevenson G, Zimmerman J, editors. Diseases of swine. NJ: Wiley Publishers; 2012. p. $841-55$.

2. le Tien HT, Nishibori T, Nishitani Y, Nomoto R, Osawa R. Reappraisal of the taxonomy of Streptococcus suis serotypes 20, 22, 26, and 33 based on DNA-DNA homology and sodA and recN phylogenies. Vet Microbiol. 2013;162:842-9.

3. Hill JE, Gottschalk M, Brousseau R, Harel J, Hemmingsen SM, Goh SH. Biochemical analysis, cpn60 and 16S rDNA sequence data indicate that Streptococcus suis serotypes 32 and 34, isolated from pigs, are Streptococcus orisratti. Vet Microbiol. 2005;107:63-9.
4. Segura M. Streptococcus suis: an emerging human threat. J Infect Dis. 2009;199:4-6.

5. Yu H, Jing $H$, Chen Z, Zheng $H$, Zhu X, Wang H, Wang S, Liu L, Zu R, Luo $L$, et al. Human Streptococcus suis outbreak, Sichuan, China. Emerg Infect Dis. 2006;12:914-20.

6. Mai NT, Hoa NT, Nga TV, le Linh D, Chau TT, Sinh DX, Phu NH, Chuong LV, Diep TS, Campbell J, et al. Streptococcus suis meningitis in adults in Vietnam. Clin Infect Dis. 2008;46:659-67.

7. King SJ, Leigh JA, Heath PJ, Luque I, Tarradas C, Dowson CG, Whatmore AM. Development of a multilocus sequence typing scheme for the pig pathogen Streptococcus suis: identification of virulent clones and potential capsular serotype exchange. J Clin Microbiol. 2002;40:3671-80.

8. Fittipaldi N, Xu JG, Lacouture S, Tharavichitkul P, Osaki M, Sekizaki T, Takamatsu D, Gottschalk M. Lineage and virulence of Streptococcus suis serotype 2 isolates from North America. Emerg Infect Dis. 2011;17:2239-44.

9. Baums CG, Valentin-Weigand P. Surface-associated and secreted factors of Streptococcus suis in epidemiology, pathogenesis and vaccine development. Anim Health Res Rev. 2009;10:65-83.

10. Fittipaldi N, Segura M, Grenier D, Gottschalk M. Virulence factors involved in the pathogenesis of the infection caused by the swine pathogen and zoonotic agent Streptococcus suis. Future Microbiol. 2012;7:259-79.

11. Charland N, Harel J, Kobisch M, Lacasse S, Gottschalk M. Streptococcus suis serotype 2 mutants deficient in capsular expression. Microbiology. 1998;144(Pt 2):325-32.

12. Smith HE, Damman M, van der Velde J, Wagenaar F, Wisselink HJ, StockhofeZurwieden N, Smits MA. Identification and characterization of the cps locus of Streptococcus suis serotype 2: the capsule protects against phagocytosis and is an important virulence factor. Infect Immun. 1999;67:1750-6.

13. Gottschalk MG, Lacouture S, Dubreuil JD. Characterization of Streptococcus suis capsular type-2 hemolysin. Microbiology. 1995;141:189-95.

14. Vecht U, Wisselink HJ, Jellema ML, Smith HE. Identification of two proteins associated with virulence of Streptococcus suis type 2 . Infect Immun. 1991:59:3156-62.

15. Hu QY, Liu P, Yu ZJ, Zhao G, Li J, Teng L, Zhou MG, Bei WC, Chen HC, Jin ML. Identification of a cell wall-associated subtilisin-like serine protease involved in the pathogenesis of Streptococcus suis serotype 2. Microb Pathogenesis. 2010;48:103-9.

16. Ge JC, Feng YJ, Ji HF, Zhang HM, Zheng F, Wang CJ, Yin ZM, Pan XZ, Tang JQ. Inactivation of dipeptidyl peptidase IV attenuates the virulence of Streptococcus suis serotype 2 that causes streptococcal toxic shock syndrome. Curr Microbiol. 2009;59:248-55.

17. de Buhr N, Neumann A, Jerjomiceva N, von Kockritz-Blickwede M, Baums CG. Streptococcus suis DNase SsnA contributes to degradation of neutrophil extracellular traps (NETs) and evasion of NET-mediated antimicrobial activity. Microbiology. 2014;160:385-95.

18. Allen AG, Lindsay H, Seilly D, Bolitho S, Peters SE, Maskell DJ. Identification and characterisation of hyaluronate lyase from Streptococcus suis. Microb Pathogenesis. 2004;36:327-35.

19. Girish KS, Kemparaju K. The magic glue hyaluronan and its eraser hyaluronidase: a biological overview. Life Sci. 2007;80:1921-43.

20. Kreil G. Hyaluronidases_a group of neglected enzymes. Protein Sci. 1995:4:1666-9.

21. Hynes WL, Walton SL. Hyaluronidases of Gram-positive bacteria. FEMS Microbiol Lett. 2000;183:201-7.

22. Pecharki D, Petersen FC, Scheie AA. Role of hyaluronidase in Streptococcus intermedius biofilm. Microbiology. 2008;154:932-8.

23. Wang Z, Guo C, Xu Y, Liu G, Lu C, Liu Y. Two novel functions of hyaluronidase from Streptococcus agalactiae are enhanced intracellular survival and inhibition of proinflammatory cytokine expression. Infect Immun. 2014:82:2615-25.

24. King SJ, Allen AG, Maskell DJ, Dowson CG, Whatmore AM. Distribution, genetic diversity, and variable expression of the gene encoding hyaluronate lyase within the Streptococcus suis population. J Bacteriol. 2004;186:4740-7.

25. Wu T, Yuan FY, Chang HT, Zhang L, Chen GP, Tan C, Chen HC, Bei WC. Identification of a novel angiogenin inhibitor 1 and its association with hyaluronidase of Streptococcus suis serotype 2. Microb Pathogenesis. 2010;49:32-7.

26. Starr CR, Engleberg NC. Role of hyaluronidase in subcutaneous spread and growth of group A streptococcus. Infect Immun. 2006;74:40-8.

27. Hynes W, Johnson C, Stokes M. A single nucleotide mutation results in loss of enzymatic activity in the hyaluronate lyase gene of Streptococcus pyogenes. Microb Pathogenesis. 2009;47:308-13. 
28. Petrey AC, de la Motte CA. Hyaluronan, a crucial regulator of inflammation. Front Immunol. 2014;5:101.

29. Smith RF, Willett NP. Rapid plate method for screening hyaluronidase and chondroitin sulfatase-producing microorganisms. Appl Microbiol. 1968;16:1434-6.

30. Untergasser A, Nijveen H, Rao X, Bisseling T, Geurts R, Leunissen JA. Primer3Plus, an enhanced web interface to Primer3. Nucleic Acids Res. 2007;35:W71-4.

31. Stins MF, Prasadarao NV, Zhou J, Arditi M, Kim KS. Bovine brain microvascular endothelial cells transfected with SV40-large T antigen: development of an immortalized cell line to study pathophysiology of CNS disease. In Vitro Cell Dev Biol Anim. 1997;33:243-7.

32. Tan C, Liu M, Jin M, Liu J, Chen Y, Wu T, Fu T, Bei W, Chen $H$. The key virulence-associated genes of Streptococcus suis type 2 are upregulated and differentially expressed in vivo. FEMS Microbiol Lett. 2008;278:108-14.

33. Vecht U, Wisselink HJ, Stockhofe-Zurwieden N, Smith HE. Characterization of virulence of the Streptococcus suis serotype 2 reference strain Henrichsen $\mathbf{S} 735$ in newborn gnotobiotic pigs. Vet Microbiol. 1996;51:125-36.
34. Cho KH, Caparon MG. Patterns of virulence gene expression differ between biofilm and tissue communities of Streptococcus pyogenes. Mol Microbiol. 2005;57:1545-56.

35. Bonifait L, Grignon L, Grenier D. Fibrinogen induces biofilm formation by Streptococcus suis and enhances its antibiotic resistance. Appl Environ Microb. 2008;74:4969-72.

36. Charland N, Nizet V, Rubens CE, Kim KS, Lacouture S, Gottschalk M. Streptococcus suis serotype 2 interactions with human brain microvascular endothelial cells. Infect Immun. 2000;68:637-43.

37. Aoki K, Matsumoto S, Hirayama Y, Wada T, Ozeki Y, Niki M, Domenech P, Umemori K, Yamamoto S, Mineda A, et al. Extracellular mycobacterial DNA-binding protein 1 participates in mycobacterium-lung epithelial cell interaction through hyaluronic acid. J Biol Chem. 2004;279:39798-806.

38. Zhang M, McDonald FM, Sturrock SS, Charnock SJ, Humphery-Smith I, Black GW. Group A streptococcus cell-associated pathogenic proteins as revealed by growth in hyaluronic acid-enriched media. Proteomics. 2007;7:1379-90.

39. Kobayashi H, Terao T. Hyaluronic acid-specific regulation of cytokines by human uterine fibroblasts. Am J Physiol. 1997;273:C1151-9.

\section{Submit your next manuscript to BioMed Central and take full advantage of:}

- Convenient online submission

- Thorough peer review

- No space constraints or color figure charges

- Immediate publication on acceptance

- Inclusion in PubMed, CAS, Scopus and Google Scholar

- Research which is freely available for redistribution

Submit your manuscript at

www.biomedcentral.com/submit

C Biomed Central 